\title{
5-Exo-dig radical cyclization of enediynes: the first synthesis of tin-substituted benzofulvenes
}

\author{
Serguei V. Kovalenko, Scott Peabody, Mariappan Manoharan, \\ Ronald J. Clark, Igor V. Alabugin* \\ Department of Chemistry and Biochemistry, Florida State University, Tallahassee,
}

Florida 32306-4390

\section{SUPPORTING INFORMATION. COMPUTATIONAL RESULTS.}

Optimized Geometries
Computed at the

UB3LYP/3-21G* Level

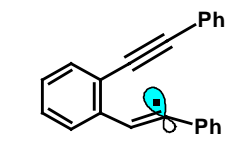

Total energy $=-842.59138$

$\begin{array}{llll}6 & 1.074951 & 0.000851 & -3.839078\end{array}$

$\begin{array}{llll}6 & 1.177212 & 0.010072 & -2.432168\end{array}$

$\begin{array}{llll}6 & 2.466124 & 0.041192 & -1.831053\end{array}$

$\begin{array}{llll}6 & 3.594906 & 0.061080 & -2.666496\end{array}$

$\begin{array}{lllll}6 & 3.477692 & 0.051520 & -4.053553\end{array}$

$\begin{array}{llll}6 & 2.210126 & 0.021350 & -4.642630\end{array}$

$\begin{array}{llll}1 & 0.087966 & -0.022928 & -4.284084\end{array}$

$1 \quad 4.578604 \quad 0.084714 \quad-2.209421$

$\begin{array}{lllll}1 & 4.367289 & 0.067535 & -4.672032\end{array}$

$\begin{array}{lllll}1 & 2.108670 & 0.013732 & -5.721309\end{array}$

$\begin{array}{lllll}6 & 2.675007 & 0.054213 & -0.371378\end{array}$

$\begin{array}{llll}6 & 1.758033 & 0.043511 & 0.567283\end{array}$

$\begin{array}{lllll}6 & -0.013177 & -0.012330 & -1.656700\end{array}$

$\begin{array}{llll}6 & -1.081316 & -0.033241 & -1.076509\end{array}$

$\begin{array}{lllll}1 & 3.730616 & 0.075820 & -0.075616\end{array}$

$\begin{array}{llll}6 & 1.263273 & 0.044078 & 1.863983\end{array}$

$\begin{array}{llll}6 & 0.979143 & -1.181416 & 2.544058\end{array}$

$\begin{array}{lllll}6 & 0.425587 & -1.169290 & 3.815703\end{array}$

$\begin{array}{llll}6 & 0.124077 & 0.043014 & 4.454285\end{array}$

$\begin{array}{llll}6 & 0.385292 & 1.255692 & 3.798880\end{array}$

$\begin{array}{llll}6 & 0.938616 & 1.268626 & 2.527333\end{array}$

$\begin{array}{lllll}1 & 1.204837 & -2.116076 & 2.045894\end{array}$

$\begin{array}{lllll}1 & 0.222391 & -2.107816 & 4.318961\end{array}$

$\begin{array}{llll}1 & -0.309108 & 0.042698 & 5.447242\end{array}$

$\begin{array}{lllll}1 & 0.150453 & 2.193749 & 4.288991\end{array}$

$\begin{array}{lllll}1 & 1.132834 & 2.203224 & 2.016088\end{array}$

$\begin{array}{llll}6 & -2.303471 & -0.058211 & -0.347588\end{array}$

$\begin{array}{llll}6 & -3.543670 & -0.049227 & -1.019230\end{array}$

$\begin{array}{llll}6 & -4.733372 & -0.073408 & -0.295781\end{array}$

$\begin{array}{llll}6 & -4.707855 & -0.106650 & 1.101852\end{array}$

$\begin{array}{llll}6 & -3.482478 & -0.115850 & 1.774813\end{array}$

$\begin{array}{llll}6 & -2.286086 & -0.092039 & 1.062812\end{array}$

$\begin{array}{llll}1 & -3.557351 & -0.023104 & -2.101833\end{array}$

$\begin{array}{llll}1 & -5.681591 & -0.066215 & -0.820780\end{array}$

$\begin{array}{llll}1 & -5.636019 & -0.125296 & 1.661192\end{array}$

$\begin{array}{llll}1 & -3.456389 & -0.141513 & 2.857911\end{array}$

$\begin{array}{llll}1 & -1.334893 & -0.099743 & 1.575759\end{array}$

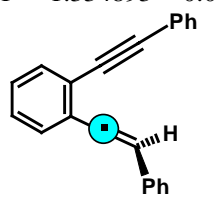

Total energy $=-842.59261$

$\begin{array}{llll}6 & 2.421824 & -3.128415 & 0.407883\end{array}$

$\begin{array}{lllll}6 & 1.730504 & -1.966687 & 0.031339\end{array}$

$\begin{array}{lllll}6 & 2.489754 & -0.776911 & -0.338082\end{array}$

$\begin{array}{lllll}6 & 3.925268 & -0.853054 & -0.275458\end{array}$

$\begin{array}{lllll}6 & 4.559942 & -2.017067 & 0.103583\end{array}$

$\begin{array}{lllll}6 & 3.813876 & -3.164395 & 0.444867\end{array}$

$\begin{array}{llll}1 & 1.845556 & -4.005322 & 0.676600\end{array}$

$\begin{array}{llll}1 & 4.493192 & 0.029794 & -0.540992\end{array}$

$\begin{array}{llll}1 & 5.642699 & -2.051485 & 0.138077\end{array}$

$\begin{array}{lllll}1 & 4.323136 & -4.073485 & 0.740141\end{array}$

$\begin{array}{lllll}6 & 1.861312 & 0.371216 & -0.722949\end{array}$

$\begin{array}{lllll}6 & 1.260007 & 1.422258 & -1.216377\end{array}$

$\begin{array}{lllll}1 & 1.105324 & 1.487962 & -2.298697\end{array}$

$\begin{array}{lllll}6 & 0.319588 & -1.923704 & 0.018891\end{array}$

$\begin{array}{llll}6 & -0.892529 & -1.830290 & 0.006710\end{array}$

$\begin{array}{llll}6 & 0.730766 & 2.574327 & -0.445687\end{array}$

$\begin{array}{lllll}6 & 0.149870 & 3.653900 & -1.130669\end{array}$

$\begin{array}{llll}6 & -0.358791 & 4.749206 & -0.431640\end{array}$

$\begin{array}{llll}6 & -0.295802 & 4.780514 & 0.962692\end{array}$

$\begin{array}{llll}6 & 0.278495 & 3.707933 & 1.654431\end{array}$

$\begin{array}{llll}6 & 0.786721 & 2.614651 & 0.958811\end{array}$

$\begin{array}{lllll}1 & 0.100985 & 3.631445 & -2.214540\end{array}$

$\begin{array}{llll}1 & -0.802781 & 5.575811 & -0.974461\end{array}$

$\begin{array}{llll}1 & -0.690771 & 5.630192 & 1.507232\end{array}$

$\begin{array}{llll}1 & 0.326448 & 3.726042 & 2.737219\end{array}$

$\begin{array}{lllll}1 & 1.228190 & 1.779624 & 1.490920\end{array}$

$\begin{array}{llll}6 & -2.303980 & -1.669499 & -0.011845\end{array}$

$\begin{array}{llll}6 & -3.164968 & -2.773438 & 0.160694\end{array}$

$\begin{array}{llll}6 & -4.545939 & -2.596307 & 0.137056\end{array}$

$\begin{array}{llll}6 & -5.091124 & -1.323545 & -0.057334\end{array}$

$\begin{array}{lllll}6 & -4.245386 & -0.222816 & -0.227439\end{array}$

$\begin{array}{llll}6 & -2.863212 & -0.387460 & -0.205888\end{array}$

$\begin{array}{llll}1 & -2.737585 & -3.757246 & 0.309643\end{array}$

$\begin{array}{llll}1 & -5.199275 & -3.450854 & 0.269840\end{array}$

$\begin{array}{llll}1 & -6.166460 & -1.190328 & -0.074927\end{array}$

$\begin{array}{lllll}1 & -4.665205 & 0.765372 & -0.375325\end{array}$

$\begin{array}{lllll}1 & -2.200625 & 0.459977 & -0.332263\end{array}$

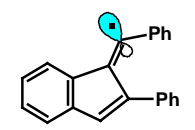

Total energy $=-842.63573$

$\begin{array}{llll}6 & -2.583463 & 0.254864 & -0.721994\end{array}$

$\begin{array}{llll}6 & -2.771826 & 0.235678 & 0.731929\end{array}$

$\begin{array}{lllll}6 & -1.499763 & 0.089195 & 1.342582\end{array}$

$\begin{array}{llll}6 & -0.479468 & 0.017532 & 0.247840\end{array}$

$\begin{array}{llll}6 & 0.821269 & -0.093936 & 0.399799\end{array}$

$\begin{array}{lllll}6 & -1.261426 & 0.131228 & -1.036172\end{array}$

$\begin{array}{llll}6 & -3.918157 & 0.326400 & 1.519046\end{array}$

$\begin{array}{llll}6 & -3.782624 & 0.274464 & 2.912998\end{array}$

$\begin{array}{llll}6 & -2.524473 & 0.133131 & 3.508567\end{array}$

$\begin{array}{llll}6 & -1.366474 & 0.038444 & 2.721439\end{array}$ $\begin{array}{llll}1 & -4.896479 & 0.436843 & 1.065039\end{array}$

$\begin{array}{llll}1 & -4.664847 & 0.345157 & 3.538862\end{array}$

$\begin{array}{llll}1 & -2.443006 & 0.095667 & 4.588581\end{array}$

$\begin{array}{llll}1 & -0.390505 & -0.072553 & 3.179980\end{array}$

$\begin{array}{lllll}1 & -3.392242 & 0.327880 & -1.434975\end{array}$

$\begin{array}{llll}6 & 2.019016 & -0.214381 & 1.085827\end{array}$

$\begin{array}{lllll}6 & 2.553654 & -1.500728 & 1.414982\end{array}$

$\begin{array}{llll}6 & 3.771803 & -1.608266 & 2.067565\end{array}$

$\begin{array}{lllll}6 & 4.504692 & -0.461969 & 2.409057\end{array}$

$\begin{array}{llll}6 & 4.001194 & 0.807353 & 2.087326\end{array}$

$\begin{array}{llll}6 & 2.786068 & 0.942377 & 1.434539\end{array}$

$\begin{array}{lllll}1 & 1.984028 & -2.383784 & 1.152989\end{array}$

$\begin{array}{lllll}1 & 4.159493 & -2.589252 & 2.317430\end{array}$

$\begin{array}{lllll}1 & 5.456495 & -0.556557 & 2.917460\end{array}$

$\begin{array}{lllll}1 & 4.567233 & 1.693390 & 2.351045\end{array}$

$\begin{array}{llll}1 & 2.395787 & 1.920105 & 1.181605\end{array}$

$\begin{array}{lllll}6 & -0.674001 & 0.122815 & -2.383154\end{array}$

$\begin{array}{lllll}6 & 0.490397 & -0.612254 & -2.673023\end{array}$

$\begin{array}{lllll}6 & 1.023705 & -0.622765 & -3.961451\end{array}$

$\begin{array}{llll}6 & 0.406035 & 0.096219 & -4.987192\end{array}$

$\begin{array}{lllll}6 & -0.750136 & 0.832401 & -4.712864\end{array}$

$\begin{array}{llll}6 & -1.281937 & 0.849853 & -3.425558\end{array}$

$\begin{array}{llll}1 & 0.965792 & -1.184380 & -1.886553\end{array}$

$\begin{array}{lllll}1 & 1.919581 & -1.197895 & -4.165734\end{array}$

$\begin{array}{llll}1 & 0.822402 & 0.087235 & -5.987731\end{array}$

$\begin{array}{llll}1 & -1.229459 & 1.402943 & -5.500252\end{array}$

$\begin{array}{llll}1 & -2.158789 & 1.449264 & -3.210266\end{array}$

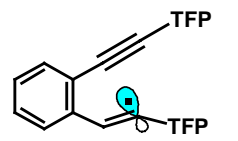

Total energy $=-1664.04333$

$\begin{array}{lllll}6 & 1.710097 & 0.000550 & -4.089933\end{array}$

$\begin{array}{lllll}6 & 1.811936 & 0.000526 & -2.682873\end{array}$

$\begin{array}{lllll}6 & 3.095943 & 0.000386 & -2.077335\end{array}$

$\begin{array}{llll}6 & 4.228749 & 0.000216 & -2.905181\end{array}$

$\begin{array}{llll}6 & 4.114162 & 0.000191 & -4.293016\end{array}$

$\begin{array}{llll}6 & 2.849176 & 0.000374 & -4.887810\end{array}$

$\begin{array}{lllll}1 & 0.723396 & 0.000701 & -4.535766\end{array}$

$\begin{array}{lllll}1 & 5.210770 & 0.000099 & -2.445370\end{array}$

$\begin{array}{llll}1 & 5.005777 & 0.000048 & -4.908147\end{array}$

$\begin{array}{lllll}1 & 2.752113 & 0.000387 & -5.966468\end{array}$

$\begin{array}{llll}6 & 3.297878 & 0.000439 & -0.617345\end{array}$

$\begin{array}{llll}6 & 2.380516 & 0.000288 & 0.312539\end{array}$

$\begin{array}{llll}6 & 0.604011 & 0.000663 & -1.935501\end{array}$

$\begin{array}{llll}6 & -0.512708 & 0.000533 & -1.460235\end{array}$

$\begin{array}{llll}1 & 4.350189 & 0.000611 & -0.312781\end{array}$

$\begin{array}{llll}6 & 1.748781 & 0.000175 & 1.530846\end{array}$

$\begin{array}{llll}6 & 1.380415 & -1.209270 & 2.174074\end{array}$

$\begin{array}{lllll}6 & 0.689397 & -1.145110 & 3.368641\end{array}$

$\begin{array}{lllll}7 & 0.364583 & -0.000091 & 3.941928\end{array}$

$\begin{array}{llll}6 & 0.688850 & 1.145048 & 3.368583\end{array}$

$\begin{array}{llll}6 & 1.379844 & 1.209477 & 2.174009\end{array}$

$\begin{array}{llll}6 & -1.836809 & 0.000108 & -0.981480\end{array}$ 
$\begin{array}{llll}6 & -2.907035 & 0.000146 & -1.887895\end{array}$

$\begin{array}{llll}6 & -4.202970 & -0.000347 & -1.391373\end{array}$

$\begin{array}{llll}7 & -4.458241 & -0.000845 & -0.098411\end{array}$

$\begin{array}{llll}6 & -3.472416 & -0.000881 & 0.776184\end{array}$

$\begin{array}{llll}6 & -2.140275 & -0.000430 & 0.384743\end{array}$

$\begin{array}{llll}9 & 0.329208 & 2.296779 & 3.993190\end{array}$

$\begin{array}{llll}9 & 0.330340 & -2.296973 & 3.993338\end{array}$

$\begin{array}{llll}9 & 1.705095 & -2.396355 & 1.600016\end{array}$

$\begin{array}{llll}9 & 1.703980 & 2.396687 & 1.599905\end{array}$

$\begin{array}{llll}9 & -1.138307 & -0.000529 & 1.305962\end{array}$

$\begin{array}{llll}9 & -3.779717 & -0.001435 & 2.095940\end{array}$

$\begin{array}{lllll}9 & -5.247857 & -0.000351 & -2.255872\end{array}$

$\begin{array}{llll}9 & -2.661841 & 0.000645 & -3.222916\end{array}$

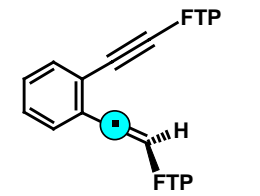

Total energy $=-1664.04907$

$\begin{array}{lllll}6 & 1.077566 & 4.545647 & 0.138538\end{array}$

$\begin{array}{lllll}6 & 0.529009 & 3.278650 & -0.103696\end{array}$

$\begin{array}{llll}6 & -0.886801 & 3.162046 & -0.434122\end{array}$

$\begin{array}{lllll}6 & -1.659135 & 4.367604 & -0.505539\end{array}$

$\begin{array}{llll}6 & -1.075424 & 5.595468 & -0.266391\end{array}$

$\begin{array}{llll}6 & 0.292128 & 5.693587 & 0.061325\end{array}$

$\begin{array}{lllll}1 & 2.130510 & 4.617003 & 0.381357\end{array}$

$\begin{array}{lllll}1 & -2.711400 & 4.289684 & -0.747138\end{array}$

$\begin{array}{lllll}1 & -1.676725 & 6.494738 & -0.327158\end{array}$

$\begin{array}{llll}1 & 0.734526 & 6.663273 & 0.251493\end{array}$

$\begin{array}{lllll}6 & -1.443676 & 1.927367 & -0.609699\end{array}$

$\begin{array}{llll}6 & -1.733063 & 0.727312 & -1.042989\end{array}$

$\begin{array}{lllll}1 & -1.500317 & 0.447567 & -2.071384\end{array}$

$\begin{array}{llll}6 & 1.311021 & 2.106256 & -0.073113\end{array}$

$\begin{array}{lllll}6 & 1.891095 & 1.040691 & -0.092063\end{array}$

$\begin{array}{llll}6 & -2.338559 & -0.347394 & -0.251412\end{array}$

$\begin{array}{llll}6 & -2.410857 & -1.629030 & -0.799989\end{array}$

$\begin{array}{llll}6 & -2.980333 & -2.649268 & -0.050746\end{array}$

$\begin{array}{lllll}7 & -3.451650 & -2.443830 & 1.163413\end{array}$

$\begin{array}{llll}6 & -3.391282 & -1.243134 & 1.703307\end{array}$

$\begin{array}{llll}6 & -2.839598 & -0.155601 & 1.036606\end{array}$

$\begin{array}{llll}6 & 2.492766 & -0.227380 & -0.147278\end{array}$

$\begin{array}{llll}6 & 3.827433 & -0.424354 & 0.230023\end{array}$

$\begin{array}{lllll}6 & 4.360826 & -1.703529 & 0.150611\end{array}$

$\begin{array}{lllll}7 & 3.653482 & -2.734802 & -0.265793\end{array}$

$\begin{array}{lllll}6 & 2.395112 & -2.578264 & -0.627302\end{array}$

$\begin{array}{lllll}6 & 1.765282 & -1.342986 & -0.588618\end{array}$

$\begin{array}{lllll}9 & -1.933604 & -1.849437 & -2.057055\end{array}$

$\begin{array}{llll}9 & -3.059787 & -3.900085 & -0.570501\end{array}$

$\begin{array}{llll}9 & -2.786873 & 1.073853 & 1.617940\end{array}$

$\begin{array}{llll}9 & -3.888912 & -1.074241 & 2.955191\end{array}$

$\begin{array}{lllll}9 & 1.704936 & -3.666847 & -1.049730\end{array}$

$\begin{array}{lllll}9 & 0.465732 & -1.201862 & -0.973446\end{array}$

$\begin{array}{lllll}9 & 4.569813 & 0.626375 & 0.662637\end{array}$

$\begin{array}{llll}9 & 5.651974 & -1.910418 & 0.511051\end{array}$

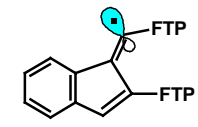

Total energy $=-1664.08994$

$\begin{array}{llll}6 & -2.411840 & 2.024469 & -0.198118\end{array}$

$\begin{array}{lllll}6 & -1.584177 & 3.226302 & -0.274978\end{array}$

$\begin{array}{llll}6 & -0.223472 & 2.833710 & -0.261816\end{array}$

$\begin{array}{lllll}6 & -0.183713 & 1.340760 & -0.170097\end{array}$

$\begin{array}{lllll}6 & 0.882149 & 0.601504 & -0.027758\end{array}$

$\begin{array}{llll}6 & -1.630829 & 0.903890 & -0.168821\end{array}$

$\begin{array}{llll}6 & -1.927911 & 4.574278 & -0.346077\end{array}$

$\begin{array}{llll}6 & -0.899442 & 5.523225 & -0.406050\end{array}$

$\begin{array}{lllll}6 & 0.443165 & 5.129083 & -0.391878\end{array}$ $\begin{array}{llll}6 & 0.794139 & 3.772519 & -0.317216\end{array}$

$\begin{array}{llll}1 & -2.966352 & 4.883973 & -0.354150\end{array}$

$\begin{array}{llll}1 & -1.146051 & 6.576621 & -0.463653\end{array}$

$\begin{array}{lllll}1 & 1.222359 & 5.880174 & -0.440045\end{array}$

$\begin{array}{lllll}1 & 1.834421 & 3.469917 & -0.306242\end{array}$

$\begin{array}{llll}1 & -3.488769 & 2.028042 & -0.171900\end{array}$

$\begin{array}{llll}6 & 2.025195 & -0.128863 & 0.119988\end{array}$

$\begin{array}{llll}6 & 2.625602 & -0.323784 & 1.394466\end{array}$

$\begin{array}{lllll}6 & 3.771735 & -1.089944 & 1.481877\end{array}$

$\begin{array}{llll}7 & 4.333880 & -1.651876 & 0.424925\end{array}$

$\begin{array}{lllll}6 & 3.801645 & -1.494952 & -0.775789\end{array}$

$\begin{array}{lllll}6 & 2.656547 & -0.752042 & -0.992004\end{array}$

$\begin{array}{llll}6 & -2.107691 & -0.474643 & -0.108410\end{array}$

$\begin{array}{lllll}6 & -3.328710 & -0.779914 & 0.511664\end{array}$

$\begin{array}{llll}6 & -3.763262 & -2.096473 & 0.562035\end{array}$

$\begin{array}{lllll}7 & -3.068551 & -3.087071 & 0.039273\end{array}$

$\begin{array}{lllll}6 & -1.925863 & -2.833091 & -0.563315\end{array}$

$\begin{array}{lllll}6 & -1.406178 & -1.548472 & -0.670363\end{array}$

$\begin{array}{lllll}9 & 4.415650 & -2.093105 & -1.828364\end{array}$

$\begin{array}{lllll}9 & 2.127375 & -0.597315 & -2.229833\end{array}$

$\begin{array}{lllll}9 & 4.355118 & -1.280535 & 2.693019\end{array}$

$\begin{array}{llll}9 & 2.059041 & 0.247751 & 2.487722\end{array}$

$\begin{array}{llll}9 & -0.232899 & -1.355269 & -1.341910\end{array}$

$\begin{array}{lllll}9 & -1.240831 & -3.869940 & -1.107163\end{array}$

$\begin{array}{llll}9 & -4.940592 & -2.386486 & 1.170475\end{array}$

$\begin{array}{llll}9 & -4.093466 & 0.211403 & 1.056340\end{array}$

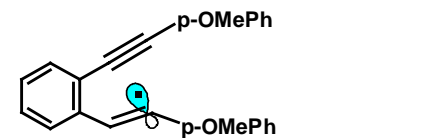

Total energy $=-1070.38341$

$\begin{array}{lllll}6 & 2.098042 & 0.106456 & -4.376824\end{array}$

$\begin{array}{llll}6 & 2.081178 & 0.097372 & -2.965880\end{array}$

$\begin{array}{lllll}6 & 3.316167 & 0.095441 & -2.258894\end{array}$

$\begin{array}{lllll}6 & 4.510933 & 0.105390 & -2.997412\end{array}$

$\begin{array}{lllll}6 & 4.511070 & 0.115077 & -4.389353\end{array}$

$\begin{array}{llll}6 & 3.296714 & 0.114838 & -5.082357\end{array}$

$\begin{array}{llll}1 & 1.151652 & 0.108040 & -4.903395\end{array}$

$\begin{array}{lllll}1 & 5.452837 & 0.104235 & -2.458730\end{array}$

$\begin{array}{lllll}1 & 5.449508 & 0.122294 & -4.931169\end{array}$

$\begin{array}{lllll}1 & 3.285626 & 0.122060 & -6.165884\end{array}$

$\begin{array}{llll}6 & 3.402114 & 0.077689 & -0.786658\end{array}$

$\begin{array}{llll}6 & 2.405142 & 0.056372 & 0.067529\end{array}$

$\begin{array}{lllll}6 & 0.829695 & 0.092769 & -2.293563\end{array}$

$\begin{array}{lllll}6 & -0.283533 & 0.095749 & -1.803862\end{array}$

$\begin{array}{llll}1 & 4.430302 & 0.079538 & -0.404667\end{array}$

$\begin{array}{llll}6 & 1.813452 & 0.022032 & 1.321387\end{array}$

$\begin{array}{llll}6 & 1.464111 & -1.213886 & 1.942433\end{array}$

$\begin{array}{llll}6 & 0.802733 & -1.245123 & 3.161575\end{array}$

$\begin{array}{lllll}6 & 0.452106 & -0.053629 & 3.812887\end{array}$

$\begin{array}{llll}6 & 0.779620 & 1.178838 & 3.213163\end{array}$

$\begin{array}{llll}6 & 1.435637 & 1.225795 & 2.001135\end{array}$

$\begin{array}{llll}1 & 1.722018 & -2.138060 & 1.440672\end{array}$

$\begin{array}{lllll}1 & 0.558247 & -2.203184 & 3.600483\end{array}$

$\begin{array}{llll}1 & 0.496203 & 2.083637 & 3.735073\end{array}$

$\begin{array}{llll}1 & 1.675571 & 2.177757 & 1.544247\end{array}$

$\begin{array}{llll}6 & -1.556353 & 0.104771 & -1.172270\end{array}$

$\begin{array}{llll}6 & -2.741029 & -0.019404 & -1.920144\end{array}$

$\begin{array}{llll}6 & -3.986948 & -0.008707 & -1.297260\end{array}$

$\begin{array}{llll}6 & -4.075425 & 0.127856 & 0.092489\end{array}$

$\begin{array}{llll}6 & -2.898057 & 0.253762 & 0.847721\end{array}$

$\begin{array}{llll}6 & -1.658627 & 0.242183 & 0.232509\end{array}$

$\begin{array}{llll}1 & -2.677407 & -0.124752 & -2.996200\end{array}$

$\begin{array}{llll}1 & -4.878596 & -0.106672 & -1.901393\end{array}$

$\begin{array}{llll}1 & -2.990389 & 0.361065 & 1.920288\end{array}$

$\begin{array}{llll}1 & -0.755025 & 0.340393 & 0.817586\end{array}$

$\begin{array}{lllll}8 & -0.211307 & 0.024791 & 5.023707\end{array}$

$\begin{array}{llll}8 & -5.257207 & 0.150023 & 0.811568\end{array}$

$\begin{array}{lllll}6 & -0.585806 & -1.221887 & 5.686425\end{array}$

$\begin{array}{lllll}1 & -1.270772 & -1.815427 & 5.069211\end{array}$ $\begin{array}{llll}1 & 0.294922 & -1.826461 & 5.933987\end{array}$

$\begin{array}{llll}1 & -1.089288 & -0.912793 & 6.602428\end{array}$

$\begin{array}{llll}6 & -6.512441 & 0.024462 & 0.076905\end{array}$

$\begin{array}{lllll}1 & -6.645981 & 0.845576 & -0.637703\end{array}$

$\begin{array}{llll}1 & -6.574004 & -0.931923 & -0.456393\end{array}$

$\begin{array}{lllll}1 & -7.290283 & 0.070861 & 0.839270\end{array}$

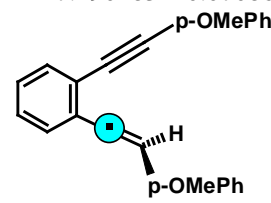

Total energy $=-1070.38416$

$\begin{array}{llll}6 & 1.083002 & 0.769943 & -4.607135\end{array}$

$\begin{array}{llll}6 & 1.060869 & 0.791285 & -3.203795\end{array}$

$\begin{array}{lllll}6 & 2.325130 & 0.810400 & -2.473936\end{array}$

$\begin{array}{llll}6 & 3.541005 & 0.783002 & -3.243261\end{array}$

$\begin{array}{lllll}6 & 3.511830 & 0.758664 & -4.621628\end{array}$

$\begin{array}{llll}6 & 2.283009 & 0.755622 & -5.314252\end{array}$

$\begin{array}{lllll}1 & 0.137687 & 0.759481 & -5.136012\end{array}$

$\begin{array}{lllll}1 & 4.481355 & 0.788378 & -2.706266\end{array}$

$\begin{array}{lllll}1 & 4.441617 & 0.743558 & -5.178563\end{array}$

$\begin{array}{lllll}1 & 2.271709 & 0.737179 & -6.397172\end{array}$

$\begin{array}{lllll}6 & 2.352445 & 0.847234 & -1.110383\end{array}$

$\begin{array}{llll}6 & 2.359416 & 1.020462 & 0.186189\end{array}$

$\begin{array}{lllll}1 & 2.385903 & 2.040944 & 0.584196\end{array}$

$\begin{array}{lllll}6 & -0.153821 & 0.780239 & -2.485840\end{array}$

$\begin{array}{llll}6 & -1.166320 & 0.753288 & -1.812430\end{array}$

$\begin{array}{llll}6 & 2.320209 & -0.040111 & 1.218468\end{array}$

$\begin{array}{llll}6 & 2.364538 & 0.305244 & 2.575004\end{array}$

$\begin{array}{llll}6 & 2.328884 & -0.669189 & 3.574111\end{array}$

$\begin{array}{llll}6 & 2.247230 & -2.020015 & 3.227628\end{array}$

$\begin{array}{lllll}6 & 2.197742 & -2.377797 & 1.869009\end{array}$

$\begin{array}{llll}6 & 2.232710 & -1.406973 & 0.883738\end{array}$

$\begin{array}{llll}1 & 2.430010 & 1.351423 & 2.855971\end{array}$

$\begin{array}{llll}1 & 2.365817 & -0.364058 & 4.611028\end{array}$

$\begin{array}{llll}1 & 2.129921 & -3.429753 & 1.624906\end{array}$

$\begin{array}{lllll}1 & 2.190110 & -1.689838 & -0.161782\end{array}$

$\begin{array}{lllll}6 & -2.311725 & 0.707341 & -0.976961\end{array}$

$\begin{array}{lllll}6 & -3.608578 & 0.888354 & -1.491286\end{array}$

$\begin{array}{lllll}6 & -4.723365 & 0.841326 & -0.657710\end{array}$

$\begin{array}{llll}6 & -4.566683 & 0.609621 & 0.713407\end{array}$

$\begin{array}{llll}6 & -3.276026 & 0.425841 & 1.237322\end{array}$

$\begin{array}{lllll}6 & -2.166797 & 0.473366 & 0.412173\end{array}$

$\begin{array}{lllll}1 & -3.735036 & 1.068250 & -2.551708\end{array}$

$\begin{array}{lllll}1 & -5.706248 & 0.986315 & -1.084920\end{array}$

$\begin{array}{llll}1 & -3.181418 & 0.245121 & 2.299784\end{array}$

$\begin{array}{lllll}1 & -1.173657 & 0.327035 & 0.818563\end{array}$

$\begin{array}{llll}8 & -5.601490 & 0.543379 & 1.628081\end{array}$

$\begin{array}{llll}8 & 2.207425 & -3.070834 & 4.128343\end{array}$

$\begin{array}{llll}6 & 2.268037 & -2.755398 & 5.551791\end{array}$

$\begin{array}{llll}1 & 2.234916 & -3.721339 & 6.056112\end{array}$

$\begin{array}{llll}1 & 3.198084 & -2.234750 & 5.810923\end{array}$

$\begin{array}{lllll}1 & 1.413176 & -2.144715 & 5.867107\end{array}$

$\begin{array}{llll}6 & -6.967275 & 0.704014 & 1.137044\end{array}$

$\begin{array}{llll}1 & -7.123166 & 1.695735 & 0.695606\end{array}$

$\begin{array}{llll}1 & -7.223109 & -0.065022 & 0.398282\end{array}$

$\begin{array}{llll}1 & -7.597949 & 0.591112 & 2.018940\end{array}$

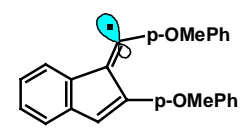

Total energy $=-1070.42780$

$\begin{array}{llll}6 & -3.201546 & -0.186871 & 0.039418\end{array}$

$\begin{array}{llll}6 & -3.156655 & -0.190819 & 1.504448\end{array}$

$\begin{array}{llll}6 & -1.796056 & -0.168290 & 1.907014\end{array}$

$\begin{array}{llll}6 & -0.958189 & -0.141536 & 0.665625\end{array}$

$\begin{array}{llll}6 & 0.355814 & -0.078164 & 0.608623\end{array}$

$\begin{array}{llll}6 & -1.938656 & -0.156543 & -0.479094\end{array}$ 
$\begin{array}{llll}6 & -4.167459 & -0.219533 & 2.463686\end{array}$

$\begin{array}{llll}6 & -3.810306 & -0.221269 & 3.818988\end{array}$

$\begin{array}{llll}6 & -2.466829 & -0.196673 & 4.208765\end{array}$

$\begin{array}{llll}6 & -1.444059 & -0.170553 & 3.247792\end{array}$

$\begin{array}{llll}1 & -5.211151 & -0.238889 & 2.170268\end{array}$

$\begin{array}{lllll}1 & -4.586070 & -0.242271 & 4.576005\end{array}$

$\begin{array}{llll}1 & -2.212944 & -0.198790 & 5.262407\end{array}$

$\begin{array}{llll}1 & -0.401964 & -0.152899 & 3.545920\end{array}$

$\begin{array}{llll}1 & -4.114495 & -0.233425 & -0.536955\end{array}$

$\begin{array}{lllll}6 & 1.615992 & -0.040810 & 1.191474\end{array}$

$\begin{array}{llll}6 & 2.328777 & -1.245885 & 1.497748\end{array}$

$\begin{array}{lllll}6 & 3.599432 & -1.198756 & 2.027791\end{array}$

$\begin{array}{llll}6 & 4.236884 & 0.033997 & 2.274002\end{array}$

$\begin{array}{llll}6 & 3.562153 & 1.226371 & 1.973154\end{array}$

$\begin{array}{llll}6 & 2.282660 & 1.195610 & 1.438982\end{array}$

$\begin{array}{lllll}1 & 1.846665 & -2.197644 & 1.313009\end{array}$

$\begin{array}{llll}1 & 4.142014 & -2.102921 & 2.270956\end{array}$

$\begin{array}{llll}1 & 4.031542 & 2.184048 & 2.153992\end{array}$

$\begin{array}{llll}1.768414 & 2.119286 & 1.205111\end{array}$

$\begin{array}{llll}6 & -1.571732 & -0.123090 & -1.899583\end{array}$

$\begin{array}{lllll}6 & -0.372197 & -0.696867 & -2.371376\end{array}$

$\begin{array}{llll}6 & -0.048843 & -0.672857 & -3.719071\end{array}$

$\begin{array}{lllll}6 & -0.910414 & -0.074397 & -4.650114\end{array}$

$\begin{array}{llll}6 & -2.101974 & 0.502956 & -4.199923\end{array}$

$\begin{array}{lllll}6 & -2.420586 & 0.478923 & -2.843418\end{array}$

$\begin{array}{lllll}6 & 0.299693 & -1.172033 & -1.668453\end{array}$

$\begin{array}{llll}1 & 0.867047 & -1.116300 & -4.086975\end{array}$

$\begin{array}{lllll}1 & -2.779896 & 0.984078 & -4.891955\end{array}$

$\begin{array}{lllll}1 & -3.331850 & 0.958246 & -2.505132\end{array}$

$\begin{array}{llll}8 & -0.491130 & -0.102685 & -5.970240\end{array}$

$\begin{array}{llll}8 & 5.508750 & -0.045464 & 2.804128\end{array}$

$\begin{array}{llll}6 & -1.355175 & 0.506423 & -6.975682\end{array}$

$\begin{array}{lllll}1 & -2.335344 & 0.015831 & -7.018061\end{array}$

$\begin{array}{lllll}1 & -1.496985 & 1.578380 & -6.791224\end{array}$

$\begin{array}{lllll}1 & -0.831881 & 0.363062 & -7.921323\end{array}$

$\begin{array}{llll}6 & 6.227796 & 1.198400 & 3.072506\end{array}$

$\begin{array}{llll}1 & 6.382594 & 1.778416 & 2.155148\end{array}$

$\begin{array}{llll}1 & 5.700751 & 1.816352 & 3.809014\end{array}$

$\begin{array}{llll}1 & 7.189897 & 0.884055 & 3.476617\end{array}$

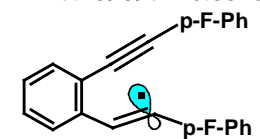

Total energy $=-1039.98990$

$6 \quad 1.689687-0.002785-4102782$

$\begin{array}{lllll}6 & 1.684427 & -0.003899 & -2.692213\end{array}$

$\begin{array}{lllll}6 & 2.923851 & -0.005048 & -1.994188\end{array}$

$\begin{array}{lllll}6 & 4.113520 & -0.005984 & -2.740430\end{array}$

$\begin{array}{lllll}6 & 4.102575 & -0.005052 & -4.132441\end{array}$

$\begin{array}{lllll}6 & 2.883454 & -0.003237 & -4.816775\end{array}$

$\begin{array}{lllll}1 & 0.739649 & -0.001969 & -4.622523\end{array}$

$\begin{array}{llll}5.059626 & -0.007056 & -2.209441\end{array}$

$\begin{array}{llll}5.036946 & -0.005601 & -4.681051\end{array}$

$\begin{array}{llll}2.864740 & -0.002443 & -5.900038\end{array}$

$\begin{array}{lllll}6 & 3.019523 & -0.004511 & -0.522872\end{array}$

$\begin{array}{llll}6 & 2.031089 & 0.002212 & 0.340659\end{array}$

$\begin{array}{lllll}6 & 0.438471 & -0.004575 & -2.009270\end{array}$

$\begin{array}{lllll}6 & -0.669577 & -0.006479 & -1.509221\end{array}$

$\begin{array}{lllll}1 & 4.049154 & -0.009718 & -0.145901\end{array}$

$\begin{array}{llll}6 & 1.454524 & 0.008286 & 1.603854\end{array}$

$\begin{array}{llll}6 & 1.096325 & -1.211623 & 2.258280\end{array}$

$\begin{array}{lllll}6 & 0.462430 & -1.194967 & 3.489470\end{array}$

$\begin{array}{llll}6 & 0.157958 & 0.021407 & 4.100008\end{array}$

$\begin{array}{lllll}6 & 0.481330 & 1.231337 & 3.486355\end{array}$

$\begin{array}{llll}6 & 1.115402 & 1.235072 & 2.255462\end{array}$

$\begin{array}{lllll}1 & 1.330406 & -2.150868 & 1.773984\end{array}$

$\begin{array}{lllll}1 & 0.195577 & -2.115618 & 3.991303\end{array}$

$\begin{array}{llll}1 & 0.228103 & 2.157218 & 3.985532\end{array}$

$\begin{array}{lll}1.363332 & 2.169295 & 1.768518\end{array}$

$\begin{array}{lllll}6 & -1.939047 & -0.008962 & -0.868796\end{array}$ $\begin{array}{llll}6 & -3.130776 & 0.034840 & -1.621794\end{array}$

$\begin{array}{lllll}6 & -4.367053 & 0.032569 & -0.986406\end{array}$

$\begin{array}{llll}6 & -4.424908 & -0.013139 & 0.402196\end{array}$

$\begin{array}{llll}6 & -3.264690 & -0.057162 & 1.167209\end{array}$

$\begin{array}{llll}6 & -2.025195 & -0.055333 & 0.539151\end{array}$

$\begin{array}{lllll}1 & -3.073241 & 0.070643 & -2.702062\end{array}$

$\begin{array}{lllll}1 & -5.287779 & 0.065964 & -1.552930\end{array}$

$\begin{array}{llll}1 & -3.341787 & -0.092378 & 2.245412\end{array}$

$\begin{array}{llll}1 & -1.114525 & -0.090324 & 1.119434\end{array}$

$\begin{array}{llll}9 & -0.472879 & 0.027746 & 5.314729\end{array}$

$\begin{array}{llll}9 & -5.643802 & -0.014962 & 1.024940\end{array}$

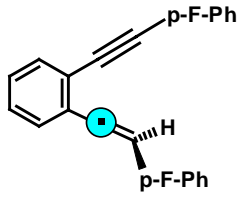

Total energy $=-1039.99133$

$\begin{array}{llll}6 & 0.772736 & 0.721909 & -4.227900\end{array}$

$\begin{array}{lllll}6 & 0.770824 & 0.724845 & -2.824520\end{array}$

$\begin{array}{lllll}6 & 2.044190 & 0.726107 & -2.112684\end{array}$

$\begin{array}{lllll}6 & 3.249884 & 0.709463 & -2.897973\end{array}$

$\begin{array}{lllll}6 & 3.201246 & 0.703856 & -4.275874\end{array}$

$\begin{array}{lllll}6 & 1.963019 & 0.711778 & -4.951425\end{array}$

$\begin{array}{llll}1 & -0.179702 & 0.724791 & -4.743688\end{array}$

$\begin{array}{lllll}1 & 4.197623 & 0.705343 & -2.374340\end{array}$

$\begin{array}{lllll}1 & 4.123056 & 0.694203 & -4.845772\end{array}$

$\begin{array}{lllll}1 & 1.936818 & 0.707728 & -6.034105\end{array}$

$\begin{array}{lllll}6 & 2.093414 & 0.730777 & -0.749323\end{array}$

$\begin{array}{llll}6 & 2.133149 & 0.870603 & 0.550238\end{array}$

$\begin{array}{lllll}1 & 2.162773 & 1.879020 & 0.976775\end{array}$

$\begin{array}{llll}6 & -0.436349 & 0.719669 & -2.093060\end{array}$

$\begin{array}{lllll}6 & -1.448632 & 0.698921 & -1.420132\end{array}$

$\begin{array}{llll}6 & 2.135184 & -0.222781 & 1.550866\end{array}$

$\begin{array}{llll}6 & 2.197973 & 0.088798 & 2.918730\end{array}$

$\begin{array}{llll}6 & 2.203873 & -0.917550 & 3.881769\end{array}$

$\begin{array}{llll}6 & 2.145951 & -2.245502 & 3.480611\end{array}$

$\begin{array}{llll}6 & 2.080563 & -2.581503 & 2.131381\end{array}$

$\begin{array}{lllll}6 & 2.074587 & -1.576082 & 1.172797\end{array}$

$\begin{array}{llll}1 & 2.245598 & 1.127193 & 3.228111\end{array}$

$\begin{array}{llll}1 & 2.253634 & -0.684212 & 4.936790\end{array}$

$\begin{array}{llll}1 & 2.033462 & -3.624864 & 1.849602\end{array}$

$\begin{array}{lllll}1 & 2.020599 & -1.826928 & 0.120007\end{array}$

$\begin{array}{llll}6 & -2.602433 & 0.665427 & -0.594190\end{array}$

$\begin{array}{lllll}6 & -3.897810 & 0.775106 & -1.142032\end{array}$

$\begin{array}{lllll}6 & -5.017313 & 0.740913 & -0.319346\end{array}$

$\begin{array}{llll}6 & -4.856535 & 0.596383 & 1.054380\end{array}$

$\begin{array}{llll}6 & -3.590995 & 0.484044 & 1.620355\end{array}$

$\begin{array}{llll}6 & -2.467448 & 0.518467 & 0.804012\end{array}$

$\begin{array}{lllll}1 & -4.011376 & 0.887765 & -2.212475\end{array}$

$\begin{array}{llll}1 & -6.015065 & 0.824747 & -0.728402\end{array}$

$\begin{array}{lllll}1 & -3.500033 & 0.368950 & 2.691981\end{array}$

$\begin{array}{llll}1 & -1.476331 & 0.427768 & 1.229769\end{array}$

$\begin{array}{lllll}9 & -5.960791 & 0.563805 & 1.861776\end{array}$

$\begin{array}{llll}9 & 2.151289 & -3.238625 & 4.423299\end{array}$

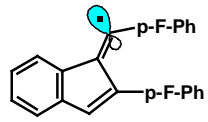

Total energy $=-1040.03421$

$\begin{array}{llll}6 & -2.963322 & -0.027355 & -0.194474\end{array}$

$\begin{array}{llll}6 & -2.950951 & -0.029848 & 1.271862\end{array}$

$\begin{array}{llll}6 & -1.600050 & -0.037088 & 1.704817\end{array}$

$\begin{array}{llll}6 & -0.734510 & -0.032507 & 0.481618\end{array}$

$\begin{array}{llll}6 & 0.579876 & 0.001156 & 0.451900\end{array}$

$\begin{array}{llll}6 & -1.689931 & -0.028581 & -0.684212\end{array}$

$\begin{array}{llll}6 & -3.984111 & -0.034078 & 2.206595\end{array}$

$\begin{array}{llll}6 & -3.657930 & -0.041408 & 3.569697\end{array}$

$\begin{array}{llll}6 & -2.323547 & -0.045859 & 3.989825\end{array}$ $\begin{array}{llll}6 & -1.278297 & -0.044195 & 3.053087\end{array}$

$\begin{array}{llll}1 & -5.020747 & -0.030389 & 1.889169\end{array}$

$\begin{array}{llll}1 & -4.450741 & -0.043576 & 4.308843\end{array}$

$\begin{array}{llll}1 & -2.093989 & -0.051396 & 5.048868\end{array}$

$\begin{array}{llll}1 & -0.243713 & -0.048943 & 3.376565\end{array}$

$\begin{array}{lllll}1 & -3.864213 & -0.052605 & -0.790849\end{array}$

$\begin{array}{llll}6 & 1.848310 & 0.020291 & 1.015616\end{array}$

$\begin{array}{llll}6 & 2.544892 & -1.193836 & 1.310329\end{array}$

$\begin{array}{llll}6 & 3.827781 & -1.163026 & 1.828584\end{array}$

$\begin{array}{lllll}6 & 4.455693 & 0.060343 & 2.060588\end{array}$

$\begin{array}{llll}6 & 3.810640 & 1.263981 & 1.777094\end{array}$

$\begin{array}{llll}6 & 2.528140 & 1.255175 & 1.257647\end{array}$

$\begin{array}{lllll}1 & 2.048417 & -2.138508 & 1.129269\end{array}$

$\begin{array}{llll}1 & 4.357130 & -2.077353 & 2.061266\end{array}$

$\begin{array}{llll}1 & 4.327660 & 2.194653 & 1.969027\end{array}$

$\begin{array}{llll}1 & 2.020029 & 2.183304 & 1.030771\end{array}$

$\begin{array}{llll}6 & -1.290147 & -0.013850 & -2.097072\end{array}$

$\begin{array}{lllll}6 & -0.105031 & -0.634586 & -2.534184\end{array}$

$\begin{array}{lllll}6 & 0.251676 & -0.628667 & -3.879479\end{array}$

$\begin{array}{lllll}6 & -0.572481 & -0.003146 & -4.806057\end{array}$

$\begin{array}{lllll}6 & -1.747563 & 0.621707 & -4.404916\end{array}$

$\begin{array}{lllll}6 & -2.100438 & 0.619349 & -3.059901\end{array}$

$\begin{array}{lllll}1 & 0.529956 & -1.133609 & -1.814230\end{array}$

$\begin{array}{lllll}1 & 1.159382 & -1.109310 & -4.218801\end{array}$

$\begin{array}{llll}1 & -2.364766 & 1.113007 & -5.145062\end{array}$

$\begin{array}{lllll}1 & -2.998985 & 1.133280 & -2.741156\end{array}$

$\begin{array}{lllll}9 & 5.723481 & 0.079889 & 2.571958\end{array}$

$\begin{array}{lllll}9 & -0.221203 & 0.003530 & -6.130036\end{array}$

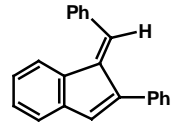

Total energy $=-843.30179$

$\begin{array}{lllll}6 & -2.514209 & -0.116889 & -0.662451\end{array}$

$\begin{array}{lllll}6 & -2.539105 & -0.118482 & 0.803213\end{array}$

$\begin{array}{lllll}6 & -1.195578 & -0.067041 & 1.277263\end{array}$

$\begin{array}{lllll}6 & -0.305898 & -0.014377 & 0.080351\end{array}$

$\begin{array}{lllll}6 & 1.030635 & 0.155802 & -0.035861\end{array}$

$\begin{array}{lllll}6 & -1.227599 & -0.048101 & -1.102578\end{array}$

$\begin{array}{llll}6 & -3.610286 & -0.129497 & 1.692063\end{array}$

$\begin{array}{lllll}6 & -3.347877 & -0.069436 & 3.066442\end{array}$

$\begin{array}{lllll}6 & -2.033934 & 0.013897 & 3.535399\end{array}$

$\begin{array}{llll}6 & -0.949990 & 0.019123 & 2.645066\end{array}$

$\begin{array}{llll}1 & -4.630661 & -0.170054 & 1.327623\end{array}$

$\begin{array}{llll}1 & -4.171122 & -0.076888 & 3.771623\end{array}$

$\begin{array}{llll}1 & -1.846621 & 0.075974 & 4.601121\end{array}$

$\begin{array}{llll}1 & 0.060805 & 0.088646 & 3.022166\end{array}$

$1 \quad-3.391154-0.189344 \quad-1.289660$

$\begin{array}{llll}6 & 2.058570 & 0.115843 & 1.017380\end{array}$

$\begin{array}{llll}6 & 2.080642 & -0.897890 & 1.993383\end{array}$

$\begin{array}{llll}6 & 3.093097 & -0.937210 & 2.951622\end{array}$

$\begin{array}{llll}6 & 4.104231 & 0.027548 & 2.948396\end{array}$

$\begin{array}{llll}6 & 4.106715 & 1.025192 & 1.969516\end{array}$

$\begin{array}{llll}6 & 3.101170 & 1.061819 & 1.005163\end{array}$

$\begin{array}{llll}1 & 1.310771 & -1.659786 & 1.979582\end{array}$

$\begin{array}{llll}1 & 3.100442 & -1.727475 & 3.693531\end{array}$

$\begin{array}{lllll}1 & 4.889991 & -0.005312 & 3.693999\end{array}$

$\begin{array}{llll}1 & 4.893951 & 1.770024 & 1.955827\end{array}$

$\begin{array}{lllll}1 & 3.106625 & 1.833179 & 0.242844\end{array}$

$\begin{array}{lllll}6 & -0.792798 & -0.027433 & -2.511663\end{array}$

$\begin{array}{lllll}6 & 0.199520 & -0.906462 & -2.985351\end{array}$

$\begin{array}{lllll}6 & 0.584886 & -0.885039 & -4.326150\end{array}$

$\begin{array}{llll}6 & -0.014337 & 0.008175 & -5.217627\end{array}$

$\begin{array}{lllll}6 & -1.004931 & 0.881213 & -4.760185\end{array}$

$\begin{array}{lllll}6 & -1.389120 & 0.866084 & -3.419821\end{array}$

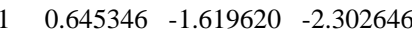

$1 \quad 1.345611 \quad-1.572940 \quad-4.677330$

$\begin{array}{lllll}1 & 0.286988 & 0.023312 & -6.258581\end{array}$

$\begin{array}{lllll}1 & -1.471267 & 1.579389 & -5.445942\end{array}$

$\begin{array}{llll}1 & -2.140340 & 1.558099 & -3.057556\end{array}$ 
$\begin{array}{llll}1 & 1.407808 & 0.351764 & -1.038011\end{array}$

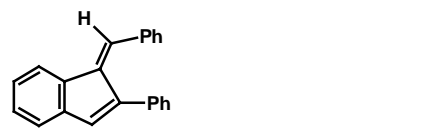

Total energy $=-843.29788$

$\begin{array}{llll}6 & -2.400519 & 0.572691 & 0.496330\end{array}$

$\begin{array}{llll}6 & -2.411746 & 0.330889 & 1.939648\end{array}$

$\begin{array}{llll}6 & -1.129289 & -0.157262 & 2.303785\end{array}$

$\begin{array}{llll}6 & -0.273706 & -0.168230 & 1.078474\end{array}$

$\begin{array}{llll}6 & 1.043690 & -0.485068 & 1.129071\end{array}$

$\begin{array}{lllll}6 & -1.173606 & 0.279260 & -0.030631\end{array}$

$\begin{array}{llll}6 & -3.414408 & 0.479128 & 2.894667\end{array}$

$\begin{array}{llll}6 & -3.132935 & 0.135776 & 4.223434\end{array}$

$\begin{array}{llll}6 & -1.868778 & -0.341432 & 4.584762\end{array}$

$\begin{array}{llll}6 & -0.854943 & -0.484233 & 3.625755\end{array}$

$\begin{array}{llll}1 & -4.393577 & 0.853837 & 2.618466\end{array}$

$\begin{array}{lllll}1 & -3.901854 & 0.241883 & 4.979956\end{array}$

$\begin{array}{llll}1 & -1.669059 & -0.604150 & 5.617080\end{array}$

$\begin{array}{llll}1 & 0.121695 & -0.849828 & 3.923959\end{array}$

$\begin{array}{lllll}1 & -3.264362 & 0.871247 & -0.080500\end{array}$

$\begin{array}{llll}6 & 2.119768 & -0.370903 & 0.139003\end{array}$

$\begin{array}{lllll}6 & 2.160724 & 0.633864 & -0.846257\end{array}$

$\begin{array}{lllll}6 & 3.242519 & 0.724976 & -1.719294\end{array}$

$\begin{array}{lllll}6 & 4.304502 & -0.179049 & -1.628893\end{array}$

$\begin{array}{lllll}6 & 4.286795 & -1.171835 & -0.645078\end{array}$

$\begin{array}{llll}6 & 3.212689 & -1.257940 & 0.236632\end{array}$

$\begin{array}{lllll}1 & 1.349124 & 1.343349 & -0.916775\end{array}$

$\begin{array}{lllll}1 & 3.258316 & 1.506344 & -2.469922\end{array}$

$\begin{array}{lllll}1 & 5.143604 & -0.103164 & -2.310640\end{array}$

$\begin{array}{lllll}1 & 5.111683 & -1.869916 & -0.561596\end{array}$

$\begin{array}{lllll}1 & 3.205165 & -2.021671 & 1.007384\end{array}$

$\begin{array}{llll}6 & -0.902121 & 0.273273 & -1.483370\end{array}$

$\begin{array}{llll}6 & -0.329236 & -0.847854 & -2.108371\end{array}$

$\begin{array}{llll}6 & -0.137887 & -0.867951 & -3.488542\end{array}$

$\begin{array}{llll}6 & -0.512285 & 0.229242 & -4.269664\end{array}$

$\begin{array}{lllll}6 & -1.082220 & 1.348862 & -3.659412\end{array}$

$\begin{array}{llll}6 & -1.276270 & 1.371430 & -2.277296\end{array}$

$\begin{array}{lllll}1 & -0.040690 & -1.699870 & -1.505911\end{array}$

$\begin{array}{llll}1 & 0.302249 & -1.740993 & -3.956484\end{array}$

$\begin{array}{lllll}1 & -0.359977 & 0.211549 & -5.342605\end{array}$

$\begin{array}{lllll}1 & -1.371579 & 2.205484 & -4.257639\end{array}$

$\begin{array}{lllll}1 & -1.705049 & 2.245050 & -1.799471\end{array}$

$\begin{array}{llll}1 & 1.389881 & -0.874955 & 2.086176\end{array}$

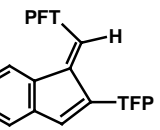

Total energy $=-1664.75705$

$\begin{array}{llll}6 & 1.461686 & 2.459062 & 0.305627\end{array}$

$\begin{array}{llll}6 & 0.139316 & 3.074187 & 0.286861\end{array}$

$\begin{array}{llll}6 & -0.820332 & 2.056821 & 0.038518\end{array}$

$\begin{array}{llll}6 & -0.087779 & 0.769795 & -0.120509\end{array}$

$\begin{array}{llll}6 & -0.561070 & -0.480733 & -0.322720\end{array}$

$\begin{array}{llll}6 & 1.369986 & 1.129111 & 0.007473\end{array}$

$\begin{array}{lllll}6 & -0.238004 & 4.405215 & 0.429054\end{array}$

$\begin{array}{lllll}6 & -1.593751 & 4.732650 & 0.300931\end{array}$

$\begin{array}{lllll}6 & -2.537805 & 3.742550 & 0.016682\end{array}$

$\begin{array}{llll}6 & -2.158657 & 2.398905 & -0.120495\end{array}$

$\begin{array}{llll}1 & 0.500908 & 5.174472 & 0.620644\end{array}$

$\begin{array}{llll}1 & -1.911194 & 5.762975 & 0.407494\end{array}$

$1 \quad-3.579789 \quad 4.014016 \quad-0.102875$

$\begin{array}{lllll}1 & -2.903583 & 1.659171 & -0.373871\end{array}$

$\begin{array}{llll}1 & 2.374523 & 2.985318 & 0.531332\end{array}$

$\begin{array}{llll}6 & -1.927320 & -0.980943 & -0.210486\end{array}$

$\begin{array}{llll}6 & -2.319693 & -2.009095 & -1.078187\end{array}$

$\begin{array}{llll}6 & -3.581973 & -2.568006 & -0.946712\end{array}$

$\begin{array}{lllll}7 & -4.427505 & -2.166801 & -0.016890\end{array}$

$\begin{array}{llll}6 & -4.078126 & -1.219085 & 0.827479\end{array}$

$\begin{array}{llll}6 & -2.834072 & -0.596825 & 0.781643\end{array}$ $\begin{array}{llll}6 & 2.528665 & 0.226537 & 0.004159\end{array}$

$\begin{array}{lllll}6 & 3.563936 & 0.410853 & 0.934397\end{array}$

$\begin{array}{llll}6 & 4.659435 & -0.441975 & 0.919260\end{array}$

$\begin{array}{lllll}7 & 4.774165 & -1.428552 & 0.052983\end{array}$

$\begin{array}{lllll}6 & 3.827290 & -1.620346 & -0.841308\end{array}$

$\begin{array}{llll}6 & 2.696101 & -0.816426 & -0.910435\end{array}$

$\begin{array}{lllll}1 & 0.136700 & -1.238385 & -0.648378\end{array}$

$\begin{array}{lllll}9 & 3.975685 & -2.634538 & -1.729779\end{array}$

$9 \begin{array}{llll}9 & 1.756146 & -1.063500 & -1.873634\end{array}$

$\begin{array}{lllll}9 & -4.969409 & -0.845084 & 1.780361\end{array}$

$\begin{array}{lllll}9 & -3.973486 & -3.554008 & -1.791413\end{array}$

$\begin{array}{lllll}9 & -1.446531 & -2.447963 & -2.026858\end{array}$

$\begin{array}{llll}5.651378 & -0.266127 & 1.827838\end{array}$

$\begin{array}{llll}9 & 3.510046 & 1.426337 & 1.843545\end{array}$

$\begin{array}{llll}9 & -2.513962 & 0.360038 & 1.692787\end{array}$

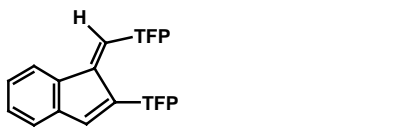

Total energy $=-1664.75582$

$\begin{array}{llll}6 & -2.720806 & -0.689992 & 1.040007\end{array}$

$\begin{array}{lllll}6 & -2.767898 & -0.409231 & 2.473730\end{array}$

$\begin{array}{llll}6 & -1.528943 & 0.173707 & 2.841778\end{array}$

$\begin{array}{llll}6 & -0.658783 & 0.205852 & 1.629420\end{array}$

$\begin{array}{llll}6 & 0.643141 & 0.556595 & 1.682955\end{array}$

$\begin{array}{llll}6 & -1.516839 & -0.304717 & 0.517182\end{array}$

$\begin{array}{llll}6 & -3.768827 & -0.613803 & 3.418007\end{array}$

$\begin{array}{lllll}6 & -3.527742 & -0.221692 & 4.742064\end{array}$

$\begin{array}{llll}6 & -2.306562 & 0.353742 & 5.105641\end{array}$

$\begin{array}{llll}6 & -1.291297 & 0.548982 & 4.155795\end{array}$

$\begin{array}{llll}1 & -4.713006 & -1.067254 & 3.140039\end{array}$

$\begin{array}{llll}1 & -4.295353 & -0.367401 & 5.492597\end{array}$

$\begin{array}{llll}1 & -2.140193 & 0.653969 & 6.133204\end{array}$

$\begin{array}{llll}1 & -0.348013 & 0.992057 & 4.455036\end{array}$

$\begin{array}{lllll}1 & -3.533124 & -1.111769 & 0.469824\end{array}$

$\begin{array}{llll}6 & 1.692135 & 0.415304 & 0.679621\end{array}$

$\begin{array}{lllll}6 & 2.698440 & 1.385206 & 0.630386\end{array}$

$\begin{array}{lllll}6 & 3.745128 & 1.232178 & -0.266603\end{array}$

$\begin{array}{lllll}7 & 3.825782 & 0.188891 & -1.069760\end{array}$

$\begin{array}{lllll}6 & 2.904863 & -0.750956 & -1.027647\end{array}$

$\begin{array}{lllll}6 & 1.815712 & -0.690498 & -0.164038\end{array}$

$\begin{array}{llll}6 & -1.236640 & -0.285250 & -0.919513\end{array}$

$\begin{array}{llll}6 & -0.570872 & 0.778128 & -1.533329\end{array}$

$\begin{array}{llll}6 & -0.372518 & 0.748663 & -2.908710\end{array}$

$\begin{array}{lllll}7 & -0.802846 & -0.240569 & -3.663225\end{array}$

$\begin{array}{lllll}6 & -1.453216 & -1.247476 & -3.113428\end{array}$

$\begin{array}{lllll}6 & -1.697592 & -1.313269 & -1.749106\end{array}$

$\begin{array}{llll}1 & 0.997979 & 0.988187 & 2.615120\end{array}$

$\begin{array}{llll}9 & -1.891757 & -2.246502 & -3.919901\end{array}$

$\begin{array}{lllll}9 & -2.399435 & -2.357916 & -1.221948\end{array}$

$\begin{array}{lllll}9 & 3.035349 & -1.818883 & -1.854321\end{array}$

$\begin{array}{lllll}9 & 4.719797 & 2.172522 & -0.330891\end{array}$

$\begin{array}{llll}9 & 2.634444 & 2.453257 & 1.473521\end{array}$

$\begin{array}{lllll}9 & 0.279154 & 1.778819 & -3.506133\end{array}$

$\begin{array}{lllll}9 & -0.135827 & 1.843698 & -0.802457\end{array}$

$\begin{array}{lllll}9 & 0.912258 & -1.708871 & -0.124557\end{array}$

PhMeOp-

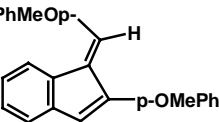

Total energy $=-1071.09330$

$\begin{array}{llll}6 & -2.987859 & -0.162222 & -0.423048\end{array}$

$\begin{array}{llll}6 & -3.021272 & -0.131536 & 1.040462\end{array}$

$\begin{array}{llll}6 & -1.680909 & -0.040046 & 1.520884\end{array}$

$\begin{array}{llll}6 & -0.786649 & 0.007945 & 0.328359\end{array}$

$\begin{array}{llll}6 & 0.548975 & 0.203594 & 0.213327\end{array}$

$\begin{array}{llll}6 & -1.699492 & -0.069318 & -0.857985\end{array}$

$\begin{array}{lllll}6 & -4.097285 & -0.145293 & 1.924289\end{array}$

$\begin{array}{llll}6 & -3.845712 & -0.045211 & 3.297972\end{array}$

$\begin{array}{llll}6 & -2.537004 & 0.083417 & 3.772135\end{array}$ $\begin{array}{llll}6 & -1.448283 & 0.090772 & 2.888118\end{array}$

$\begin{array}{llll}1 & -5.114206 & -0.217233 & 1.554803\end{array}$

$\begin{array}{llll}1 & -4.673137 & -0.054643 & 3.998392\end{array}$

$\begin{array}{llll}1 & -2.358185 & 0.180682 & 4.836812\end{array}$

$\begin{array}{llll}1 & -0.442634 & 0.199951 & 3.269751\end{array}$

$1-3.858186 \quad-0.273946 \quad-1.053663$

$\begin{array}{llll}6 & 1.582951 & 0.173429 & 1.252888\end{array}$

$\begin{array}{llll}6 & 1.590985 & -0.785405 & 2.279406\end{array}$

$\begin{array}{llll}6 & 2.613301 & -0.823739 & 3.226611\end{array}$

$\begin{array}{lllll}6 & 3.663551 & 0.098550 & 3.165036\end{array}$

$\begin{array}{llll}6 & 3.684370 & 1.043777 & 2.126364\end{array}$

$\begin{array}{llll}6 & 2.671756 & 1.070582 & 1.182598\end{array}$

$\begin{array}{llll}1 & 0.798603 & -1.522692 & 2.319388\end{array}$

$\begin{array}{llll}1 & 2.589690 & -1.583240 & 3.996180\end{array}$

$\begin{array}{llll}1 & 4.512902 & 1.738443 & 2.088855\end{array}$

$\begin{array}{lllll}1 & 2.703272 & 1.801993 & 0.382617\end{array}$

$\begin{array}{llll}6 & -1.259057 & -0.066181 & -2.263967\end{array}$

$\begin{array}{lllll}6 & -0.231965 & -0.918282 & -2.722615\end{array}$

$\begin{array}{lllll}6 & 0.150642 & -0.921075 & -4.055861\end{array}$

$\begin{array}{llll}6 & -0.480593 & -0.076466 & -4.981349\end{array}$

$\begin{array}{lllll}6 & -1.503512 & 0.769945 & -4.543377\end{array}$

$\begin{array}{llll}6 & -1.882153 & 0.771974 & -3.200650\end{array}$

$\begin{array}{lllll}1 & 0.244078 & -1.597190 & -2.025920\end{array}$

$\begin{array}{lllll}1 & 0.930516 & -1.577908 & -4.418205\end{array}$

$\begin{array}{lllll}1 & -2.003842 & 1.435790 & -5.233478\end{array}$

$\begin{array}{lllll}1 & -2.660968 & 1.446564 & -2.864614\end{array}$

$\begin{array}{lllll}1 & 0.917367 & 0.408055 & -0.790710\end{array}$

$\begin{array}{llll}8 & -0.021233 & -0.159020 & -6.285656\end{array}$

$\begin{array}{lllll}8 & 4.721822 & 0.155404 & 4.051956\end{array}$

$\begin{array}{llll}6 & 4.753296 & -0.805674 & 5.151497\end{array}$

$\begin{array}{llll}1 & 3.875423 & -0.703260 & 5.800518\end{array}$

$\begin{array}{lllll}1 & 4.813628 & -1.837119 & 4.784100\end{array}$

$\begin{array}{llll}1 & 5.655880 & -0.561619 & 5.711695\end{array}$

$\begin{array}{lllll}6 & -0.654839 & 0.688780 & -7.290198\end{array}$

$\begin{array}{lllll}1 & -1.724574 & 0.467531 & -7.389469\end{array}$

$\begin{array}{lllll}1 & -0.527147 & 1.753655 & -7.060008\end{array}$

$\begin{array}{lllll}1 & -0.142671 & 0.451206 & -8.222825\end{array}$

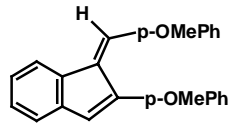

Total energy $=-1071.08956$

$\begin{array}{llll}6 & -2.956443 & -0.152637 & 1.269096\end{array}$

$\begin{array}{llll}6 & -2.904691 & -0.085928 & 2.728628\end{array}$

$\begin{array}{lllll}6 & -1.536230 & -0.067051 & 3.109818\end{array}$

$\begin{array}{llll}6 & -0.709952 & -0.058096 & 1.865685\end{array}$

$\begin{array}{llll}6 & 0.641652 & 0.073489 & 1.911307\end{array}$

$\begin{array}{llll}6 & -1.693190 & -0.149562 & 0.743019\end{array}$

$\begin{array}{llll}6 & -3.911430 & -0.067570 & 3.691553\end{array}$

$\begin{array}{llll}6 & -3.548961 & -0.031316 & 5.043917\end{array}$

$\begin{array}{llll}6 & -2.201883 & -0.008414 & 5.421217\end{array}$

$\begin{array}{lllll}6 & -1.186393 & -0.018744 & 4.453995\end{array}$

$\begin{array}{llll}1 & -4.956320 & -0.079793 & 3.402069\end{array}$

$\begin{array}{lllll}1 & -4.319261 & -0.019439 & 5.806516\end{array}$

$\begin{array}{llll}1 & -1.939155 & 0.018000 & 6.472448\end{array}$

$\begin{array}{lllll}1 & -0.147167 & 0.006361 & 4.763876\end{array}$

$\begin{array}{llll}1 & -3.863891 & -0.266927 & 0.693286\end{array}$

$\begin{array}{lllll}6 & 1.650829 & 0.331358 & 0.886928\end{array}$

$\begin{array}{llll}6 & 1.394918 & 1.005683 & -0.328616\end{array}$

$\begin{array}{lllll}6 & 2.416423 & 1.271415 & -1.222983\end{array}$

$\begin{array}{llll}6 & 3.735667 & 0.875659 & -0.947817\end{array}$

$\begin{array}{llll}6 & 4.013517 & 0.222945 & 0.258289\end{array}$

$\begin{array}{lllll}6 & 2.984701 & -0.029267 & 1.160970\end{array}$

$\begin{array}{lllll}1 & 0.389596 & 1.324458 & -0.559103\end{array}$

$\begin{array}{lllll}1 & 2.227214 & 1.791586 & -2.152344\end{array}$

$\begin{array}{llll}1 & 5.021299 & -0.082392 & 0.504686\end{array}$

$\begin{array}{lllll}1 & 3.214300 & -0.525347 & 2.098380\end{array}$

$\begin{array}{llll}6 & -1.416008 & -0.365389 & -0.690862\end{array}$

$\begin{array}{lllll}6 & -0.509345 & -1.356672 & -1.118050\end{array}$

$6 \begin{array}{llll}6 & -0.303175 & -1.598201 & -2.466400\end{array}$ 


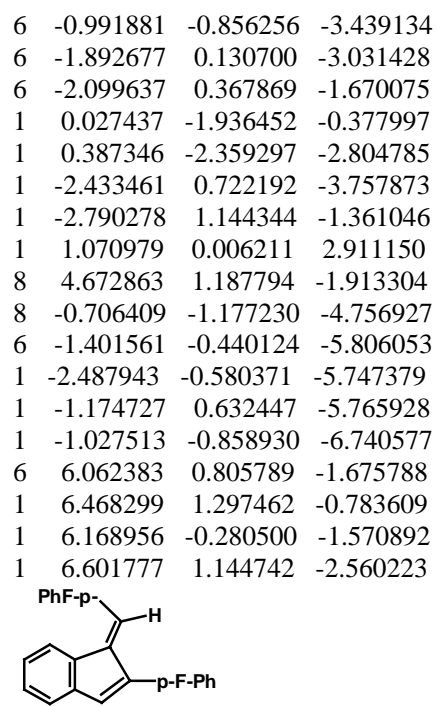

Total energy $=-1040.70036$

$\begin{array}{llll}6 & -2.793196 & -0.023578 & -0.485513\end{array}$

$\begin{array}{llll}6 & -2.796854 & -0.021775 & 0.980116\end{array}$

$\begin{array}{llll}6 & -1.445599 & -0.008578 & 1.434859\end{array}$

$\begin{array}{llll}6 & -0.572229 & 0.015805 & 0.225148\end{array}$

$\begin{array}{llll}6 & 0.767686 & 0.140932 & 0.086627\end{array}$

$\begin{array}{llll}6 & -1.511536 & 0.008589 & -0.943843\end{array}$

$\begin{array}{llll}6 & -3.855446 & 0.000479 & 1.883358\end{array}$

$\begin{array}{llll}6 & -3.572484 & 0.056517 & 3.253845\end{array}$

$\begin{array}{llll}6 & -2.250463 & 0.104922 & 3.704274\end{array}$

$\begin{array}{llll}6 & -1.179310 & 0.076750 & 2.799065\end{array}$

$\begin{array}{llll}1 & -4.881443 & -0.011248 & 1.533129\end{array}$

$\begin{array}{llll}1 & -4.385638 & 0.074319 & 3.970344\end{array}$

$\begin{array}{llll}1 & -2.046591 & 0.166338 & 4.766930\end{array}$

$\begin{array}{llll}1 & -0.162651 & 0.122595 & 3.163800\end{array}$

$\begin{array}{llll}1 & -3.680740 & -0.073262 & -1.099937\end{array}$

$\begin{array}{llll}6 & 1.813268 & 0.055314 & 1.116629\end{array}$

$\begin{array}{llll}6 & 1.802893 & -0.943225 & 2.108995\end{array}$

$\begin{array}{lllll}6 & 2.831196 & -1.030529 & 3.042651\end{array}$

$\begin{array}{llll}6 & 3.885440 & -0.126055 & 2.989631\end{array}$

$\begin{array}{lllll}6 & 3.936011 & 0.854174 & 2.005143\end{array}$

$\begin{array}{llll}6 & 2.911927 & 0.935112 & 1.067560\end{array}$

$\begin{array}{lllll}1 & 0.993065 & -1.661470 & 2.128727\end{array}$

$\begin{array}{lllll}1 & 2.832993 & -1.799925 & 3.802983\end{array}$

$\begin{array}{llll}1 & 4.774197 & 1.537260 & 1.980988\end{array}$

$\begin{array}{lllll}1 & 2.949694 & 1.694495 & 0.295034\end{array}$

$\begin{array}{llll}6 & -1.097568 & 0.015513 & -2.358613\end{array}$

$\begin{array}{llll}6 & -0.145084 & -0.896133 & -2.851822\end{array}$

$\begin{array}{lllll}6 & 0.217338 & -0.890793 & -4.196588\end{array}$

$\begin{array}{lllll}6 & -0.371751 & 0.022120 & -5.062472\end{array}$

$\begin{array}{llll}6 & -1.318649 & 0.930210 & -4.604475\end{array}$

$\begin{array}{lllll}6 & -1.677003 & 0.927423 & -3.259406\end{array}$

$\begin{array}{lllll}1 & 0.289972 & -1.625484 & -2.180279\end{array}$

$\begin{array}{lllll}1 & 0.942120 & -1.594444 & -4.583545\end{array}$

$\begin{array}{llll}1 & -1.757032 & 1.633129 & -5.300093\end{array}$

$\begin{array}{lllll}1 & -2.397913 & 1.646082 & -2.889367\end{array}$

$\begin{array}{llll}1 & 1.133025 & 0.330455 & -0.921118\end{array}$

$\begin{array}{lllll}9 & 4.894903 & -0.211383 & 3.909376\end{array}$

$\begin{array}{llll}9 & -0.014299 & 0.027159 & -6.384777\end{array}$

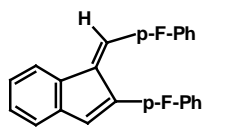

Total energy $=-1040.69695$

$\begin{array}{llll}6 & -2.728651 & -0.146764 & 0.808889\end{array}$

$\begin{array}{llll}6 & -2.730964 & -0.146553 & 2.272366\end{array}$

$\begin{array}{llll}6 & -1.378678 & -0.152553 & 2.704714\end{array}$

$\begin{array}{llll}6 & -0.506157 & -0.093886 & 1.493513\end{array}$ $\begin{array}{llll}6 & 0.841968 & 0.020254 & 1.584209\end{array}$

$\begin{array}{lllll}6 & -1.447886 & -0.127174 & 0.330898\end{array}$

$\begin{array}{llll}6 & -3.773389 & -0.163255 & 3.195556\end{array}$

$\begin{array}{lllll}6 & -3.461063 & -0.186842 & 4.561061\end{array}$

$\begin{array}{llll}6 & -2.129378 & -0.188139 & 4.988870\end{array}$

$\begin{array}{llll}6 & -1.077757 & -0.163547 & 4.060813\end{array}$

$\begin{array}{llll}1 & -4.806591 & -0.156389 & 2.867222\end{array}$

$\begin{array}{llll}1 & -4.259176 & -0.202670 & 5.294168\end{array}$

$\begin{array}{lllll}1 & -1.906315 & -0.208121 & 6.049201\end{array}$

$\begin{array}{llll}1 & -0.050851 & -0.158034 & 4.409734\end{array}$

$\begin{array}{llll}1 & -3.615352 & -0.227231 & 0.196130\end{array}$

$\begin{array}{lllll}6 & 1.866072 & 0.309478 & 0.576770\end{array}$

$\begin{array}{lllll}6 & 1.635222 & 1.123655 & -0.549307\end{array}$

$\begin{array}{lllll}6 & 2.661703 & 1.412369 & -1.440945\end{array}$

$\begin{array}{lllll}6 & 3.932581 & 0.893763 & -1.221434\end{array}$

$\begin{array}{lllll}6 & 4.198338 & 0.102967 & -0.109309\end{array}$

$\begin{array}{llll}6 & 3.174104 & -0.174913 & 0.788002\end{array}$

$1 \quad 0.651834 \quad 1.538123 \quad-0.714567$

$\begin{array}{llll}2.489274 & 2.040529 & -2.304205\end{array}$

$\begin{array}{lll}5.199142 & -0.277112 & 0.044280\end{array}$

$\begin{array}{lll}3.379384 & -0.782436 & 1.662420\end{array}$

$\begin{array}{llll}6 & -1.117542 & -0.281014 & -1.100392\end{array}$

$\begin{array}{lllll}6 & -0.194675 & -1.249574 & -1.533270\end{array}$

$\begin{array}{lllll}6 & 0.063375 & -1.430975 & -2.887982\end{array}$

$\begin{array}{lllll}6 & -0.597209 & -0.644782 & -3.825002\end{array}$

$\begin{array}{lllll}6 & -1.513854 & 0.319320 & -3.426313\end{array}$

$\begin{array}{lllll}6 & -1.772600 & 0.499190 & -2.068792\end{array}$

$\begin{array}{lllll}1 & 0.314671 & -1.861407 & -0.800388\end{array}$

$\begin{array}{llll}1 & 0.769320 & -2.176641 & -3.227937\end{array}$

$\begin{array}{lllll}1 & -2.010601 & 0.920282 & -4.176165\end{array}$

$\begin{array}{llll}1 & -2.475798 & 1.258750 & -1.748466\end{array}$

$\begin{array}{lll}1.251904 & -0.093028 & 2.587576\end{array}$

$\begin{array}{llll}4.939822 & 1.177921 & -2.102462\end{array}$

$\begin{array}{llll}-0.338712 & -0.821896 & -5.158811\end{array}$ 